\title{
A DIFFERENTIAL MEDIUM FOR THE ENUMERATION OF THE SPOILAGE YEAST ZYGOSACCHAROMYCES BAILII IN WINE
}

D. Schuller, M. Côrte- Real* and C. Leão

Centro de Ciências do Ambiente - Departamento de Biologia, Universidade do Minho, 4710-057 Braga, Portugal.

Short running title: Differential medium for Zygosaccharomyces bailii

Key words:Zygosaccharomyces bailii, spoilage yeasts, differential medium

Manuela Côrte-Real*

Phone: $351-253-604314$

Fax: $351-253-678980$

e-mail: mcortereal@bio.uminho.pt 


\begin{abstract}
A collection of yeasts, isolated mostly from spoiled wines, was used in order to develop a differential medium for Zygosaccharomyces bailii. The 118 selected strains of 21 species differed in their origin and resistance to preservatives and belonged to the genera Pichia, Torulaspora, Dekkera, Debaryomyces, Saccharomycodes, Issatchenkia, Kluyveromyces, Kloeckera, Lodderomyces, Schizosaccharomyces, Rhodotorula, Saccharomyces and Zygosaccharomyces. The design of the culture medium was based on the different ability of the various yeast species to grow in a mineral medium with glucose and formic acid (mixedsubstrate medium) as the only carbon and energy sources and supplemented with an acid-base indicator. By manipulating the concentration of the acid and the sugar it was possible to select conditions where only Z. bailii strains gave rise to alkalization, associated with a color change of the medium (positive response). The final composition of the mixed medium was adjusted as a compromise between the percentage of recovery and selectivity for Z. bailii. This was accomplished by the use of pure or mixed cultures of the yeast strains and applying the membrane filtration methodology. The microbiological analysis of two samples of contaminated "Vinho Verde" showed that the new medium can be considered as a differential medium to distinguish Z. bailii from other contaminating yeasts, having potential application in the microbiological control of wines and probably other beverages and foods.
\end{abstract}


The design of differential or selective media for preservative resistant yeasts is an ongoing concern in the quality control of foods and beverages. This is particularly relevant for the case of Zygosaccharomyces bailii, which is a commonly encountered spoilage yeast. In the food and beverages industries this species is responsible for considerable economic losses $(3,7)$. The low permeability of $Z$. bailii to weak acid preservatives at low $\mathrm{pH}$ values and its ability to metabolize acid compounds, even in the presence of glucose, are some of the physiological traits associated to its high tolerance to acidic environments $(2,5,6)$.

The aim of this work was to develop a differential medium for Z. bailii. The strategy followed was based on the use of a medium containing a mixture of glucose and formic acid, as sole carbon and energy sources with the incorporation of an acid-base indicator.

\section{Materials and Methods}

Yeast strains and culture media. The yeast strains selected for the present study (Table 1) were mostly isolated from spoiled wines and differed in their origin and resistance to preservatives. Cultures were maintained at room temperature on Yeast Extract Peptone Dextrose (YEPD) agar slants containing yeast extract $(0.5 \%, \mathrm{w} / \mathrm{v})$, peptone $(1 \%, \mathrm{w} / \mathrm{v})$, glucose $(2 \%, \mathrm{w} / \mathrm{v})$ and agar $(2 \%, \mathrm{w} / \mathrm{v}), \mathrm{pH}$ 6.9. The new Zygosaccharomyces bailii differential (ZBD) medium was based on a mineral medium supplemented with vitamins and oligoelements (8), bromocresol green as acid-base indicator $(0.005 \%, \mathrm{w} / \mathrm{v})$ and a mixture of formic acid and glucose (mixed-substrate medium). The basic mineral medium containing the acid-base indicator was adjusted to $\mathrm{pH} 4.5$, sterilized $\left(121^{\circ} \mathrm{C}\right.$ for $15 \mathrm{~min}$.) and cooled to 50 $52^{\circ} \mathrm{C}$. Subsequently, the filter-sterilized oligoelements, vitamins and substrates, previously adjusted to $\mathrm{pH} 4.5(\mathrm{NaOH} 10 \mathrm{M})$ were added. A solid medium was prepared by the addition of $\operatorname{agar}(2.0 \%, \mathrm{w} / \mathrm{v})$ to the basic mineral medium before sterilization.

Zygosaccharomyces bailii selective agar (ZBA, pH 4.0) was prepared according to Erickson (1), following the correction of the published formula, as mentioned by Makdesi and 
Beuchat (4). The Wallerstein Laboratory Nutrient (WLN, pH 5.5) and Differential (WLD, pH 5.5) Agars, both commercialized by Difco, were used for the "field trial" assays with contaminated wine samples.

Development of the mixed-substrate culture medium. After 2 days of growth on YEPD slants, a cell suspension in sterile deionized water was prepared in order to obtain an optical density $\left(\mathrm{OD}_{640}\right)$ between 0.7 and 1.0. Microplate wells containing $225 \mu 1$ of mixed-substrate liquid culture medium were inoculated with $25 \mu \mathrm{l}$ of the cell suspension. The inoculation of the solidified medium was performed by dropping $10 \mu \mathrm{l}$ of the cell suspension onto the surface of an agar plate. The microplates were mechanically shaken at $180 \mathrm{rpm}$. Color change of the drops or suspensions was evaluated after incubation at $30^{\circ} \mathrm{C}$ for $48 \mathrm{~h}$.

Validation of the culture medium - recovery of Zygosaccharomyces bailii cells. The ZBD medium, containing $0.1 \%(\mathrm{w} / \mathrm{v})$ glucose and $0.2,0.3,0.4$ or $0.5 \%(\mathrm{v} / \mathrm{v})$ formic acid, was compared with the formerly described "Z. bailii selective medium" (ZBA) (1) for the ability to recover Z. bailii cells. YEPD was used as a non-selective control medium for evaluating the percentage recovery. In order to simulate the yeast flora of a spoiled wine, several typical wine contaminating yeast species as Z. bailii, Saccharomyces cerevisiae, Pichia membranaefaciens and Dekkera anomala, were selected. These species were tested in pure or mixed cultures. The cell suspensions were obtained as described above and mixed cultures were prepared by mixing in equal proportions, Z. bailii IGC 4806 and S. cerevisiae IGC 4072; Z. bailii IGC 4806, P. membranaefaciens IGC 2487 and D. anomala IGC 5133. Decimal dilutions of these suspensions were prepared in sterile deionized water. $0.1 \mathrm{ml}$ of the selected dilutions were filtered, in triplicate, through membrane filters with $0.45 \mu \mathrm{m}$ pore size (Millipore) with the aid of partial vacuum. In order to obtain an uniform distribution of cells on the surface of the membrane, $50 \mathrm{ml}$ of sterile, deionized water were added to the inoculum $(0.1 \mathrm{ml})$ in the funnel before filtration. The filters were placed onto the surface of plates 
containing the mentioned culture media and were incubated at $30^{\circ} \mathrm{C}$ for $96 \mathrm{~h}$ before colonies were counted.

Utilization of the ZBD medium for the analysis of the yeast flora in contaminated wines. Two samples of contaminated "Vinho Verde" were collected from two containers of a wine cellar in the North of Portugal. Both samples displayed turbidity, apparently due to a refermentation process. Different volumes $(0.1,1.0$ and $5.0 \mathrm{ml})$ of each sample were filtered through a $0.45 \mu \mathrm{m}$ pore size membrane filter (Millipore) with the application of partial vacuum. In order to obtain an uniform distribution of cells on the surface of the membrane filters, the samples were diluted into $50 \mathrm{ml}$ of sterile deionized water. Membrane duplicates were placed on the surface of YEPD, WLN, WLD, and ZBD medium containing $0.1 \%(\mathrm{w} / \mathrm{v})$ glucose and $0.4 \%$ (v/v) formic acid, and incubated for $96 \mathrm{~h}$ at $30^{\circ} \mathrm{C}$. After a 4 month refrigeration period at $4^{\circ} \mathrm{C}$ the two samples of contaminated "Vinho Verde" were analyzed again by the same procedure.

\section{Results}

Design of a culture medium for the detection of Zygosaccharomyces bailii. The design of the culture medium was based on previous studies demonstrating the ability of Zygosaccharomyces bailii to use acetic acid in the presence of glucose (6). Thus, the different ability of the various yeast species to grow in a mineral medium containing a sugar and formic acid (mixed-substrate medium) as the only carbon and energy sources, was explored. The utilization of the acid is associated with alkalization; therefore the incorporation of bromocresol green as an acid-base indicator allowed the visualization of the different consumption patterns. A species exhibiting a higher acid tolerance and/or consumption rate will lead to a higher alkalization of the culture medium and, hence, to a color change from green to blue. In this sense, non-Z. bailii strains which changed the color of the medium from green to blue were considered as giving a false positive result. 
In order to define experimental conditions where only Z. bailii strains would give a positive response, the acid concentration was adjusted to $0.3,0.4$ or $0.5 \%(\mathrm{v} / \mathrm{v})$, while the glucose concentration was maintained at $0.1 \%(\mathrm{w} / \mathrm{v})$. The assays were performed in liquid or solid medium using microplate wells and agar plates, respectively, as summarized in Table 2. Almost all Z. bailii strains displayed alkalization of the mixed culture media after about $48 \mathrm{~h}$ incubation. However, three strains (ISA 1025, ISA 1095 and IGC T5167) changed the color of the medium only after an additional incubation time, which was related with the increase in the formic acid concentration. Therefore, they were considered as displaying a slow response.

No major differences were detected in the results from liquid or solid medium, since similar patterns in color change were obtained. All Zygosaccharomyces bisporus strains tested were also able to change the color of the mixed culture medium containing $0.3 \%(\mathrm{v} / \mathrm{v})$ formic acid. An increase in the acid concentration to $0.4 \%(\mathrm{v} / \mathrm{v})$ resulted in a prolonged response time $(120 \mathrm{~h})$ for three strains of Z. bisporus. Two strains of Z. rouxii (IGC 4194 and ISA 1220) exhibited false positive results in the mixed-substrate medium containing $0.3 \%$ (v/v) formic acid. However, the increase in the acid concentration to $0.5 \%(\mathrm{v} / \mathrm{v})$ eliminated those responses, even after a prolonged incubation time $(120 \mathrm{~h})$. All the other yeast species were unable to increase the $\mathrm{pH}$ of the culture media and, therefore, to change the color of the medium.

Validation of the culture medium - recovery of Zygosaccharomyces bailii cells. The applicability of the ZBD medium for the microbiological control of wines was evaluated by estimating the percentage recovery of $Z$. bailii and other typical wine contamination yeasts. In addition, the ZBD medium was compared with Zygosaccharomyces bailii selective agar (ZBA) described by Erickson (1).

As shown in Table 3, the percentage recovery of Z. bailii cells in the ZBD medium with $0.2,0.3$ or $0.4 \%(\mathrm{v} / \mathrm{v})$ formic acid and in ZBA, did not differ significantly $(\mathrm{p} \leq 0.05)$ from the value obtained with the reference culture medium (YEPD). The increase in the acid 
concentration up to $0.5 \%(\mathrm{v} / \mathrm{v})$ caused a significant decrease $(\mathrm{p} \leq 0.05)$ in the number of cells recovered compared with the YEPD medium. Contamination of wine with Z. bailii can be associated with other yeast species like S. cerevisiae, P. membranaefaciens or D. anomala. Mixed cultures of these species and Z. bailii (in equal proportions) were tested in order to simulate a contaminated wine and to determine if the presence of other contaminating yeasts could change the percentage recovery of Z. bailii. The results (Table 3 ) showed that with mixed cultures of Z. bailii with S. cerevisiae or Z. bailii with P. membranaefaciens and $D$. anomala, the percentage of recovery of $Z$. bailii for the various culture media was very similar to the values obtained for this yeast in pure culture. S. cerevisiae, when tested in pure culture, grew to a significantly lesser extent on the mixed-substrate medium, when compared to the reference medium YEPD. The percentage recovery of about $30 \%$ in the medium containing $0.2 \%(\mathrm{v} / \mathrm{v})$ formic acid dramatically decreased to less than $0.002 \%$ when the concentration of formic acid was increased to $0.4 \%(\mathrm{v} / \mathrm{v})$. A similar effect was observed with P. membranaefaciens. These results are consistent with the higher selectivity of the medium with increasing concentrations of formic acid. D. anomala was unable to grow on any of the tested culture media. The percentage recovery of S. cerevisiae obtained on ZBD containing glucose $(0.1 \%, \mathrm{w} / \mathrm{v})$ and formic acid $(0.2 \%, \mathrm{v} / \mathrm{v})$ was higher than on the ZBA medium. With the increase of the acid concentration to $0,4 \%(\mathrm{v} / \mathrm{v})$ in the presence of glucose $(0,1 \%, \mathrm{w} / \mathrm{v})$, the recovery rate decreased, being below the value determined for ZBA. The "ideal" mixedsubstrate culture medium composition for the enumeration of Z. bailii cells in the presence of other wine contaminating yeasts appeared to be this latter one.

The color and colony morphology of the four yeasts in mixed-substrate medium containing $0.2 \%(\mathrm{v} / \mathrm{v})$ formic acid is illustrated in Figure 1 . The colonies of Z. bailii are easily identified by their blue color (Fig. 1, A). On the same medium, S. cerevisiae formed white, light-green or green colonies (Fig. 1, B), indicating that the acid-base indicator, bromocresol green, might be incorporated into some cells. P. membranaefaciens formed dark green colonies (Fig. 1, C). 
Using the mixed cultures, it was possible to distinguish the blue Z. bailii colonies from the white S. cerevisiae colonies (Fig 1 D). Nevertheless, several colonies were light blue, resulting possibly from $S$. cerevisiae cells which were able to incorporate the acid-base indicator (of blue color due to the alkalization performed by Z. bailii ), as it was already observed for this yeast when tested in pure culture. P. membranaefaciens formed dark blue colonies in the presence of Z. bailii (Fig. 1 E), which could be distinguished clearly from each other by size and intensity of the blue color.

“Field trial" evaluation with the mixed-substrate mineral medium. In order to validate the differential medium developed, two samples of contaminated "Vinho Verde" from a wine cellar in the north of Portugal were analyzed by membrane filtration. The number and morphology of the colonies developed on the mixed-substrate medium were compared to other culture media such as WLN and WLD, which are commonly used for the microbiological analysis of wines. The results obtained are shown in Table 4 . The values refer to a first analysis, performed immediately after sampling, and a second analysis, after refrigeration of the wine for four months. Before refrigeration, the number of yeasts able to grow on WLN (the medium normally used for the detection of the total yeast flora in wines) was of the same order of magnitude in the two wines. Major differences were detected, using either WLD (the medium for the enumeration of the non-fermenting flora in wines as well as lactic and acetic acid bacteria) or the ZBD medium containing formic acid $(0.4 \% \mathrm{v} / \mathrm{v})$ and glucose $(0.1 \% \mathrm{w} / \mathrm{v})$. The number of yeasts able to grow on WLD was 20 fold higher in wine 2 compared with wine 1, suggesting differences in the composition of the yeast population in the two wines. In wine 2, the ZBD medium allowed the detection of 170 blue colored colonies, indicating the presence of Z. bailii. One of those blue colonies was confirmed to be Z. bailii by molecular typing. On the same medium, a similar number of slightly yellowcreamy colonies was found in both wines, but they were non-Z. bailii species when assayed by molecular typing. 
After a four months refrigeration, both wines had a similar number of yeasts able to grow on YEPD. The refrigeration period lead to a dramatic decrease in the yeast counts in WLN, resulting in $10 \%$ of the initial count for wine 1 , and $0.3 \%$ for wine 2 . No yeasts were able to grow on the mixed-substrate medium (ZBD) after refrigeration.

\section{Discussion}

All 15 strains of Zygosaccharomyces bailii tested were able to increase the $\mathrm{pH}$ of the ZBD medium containing formic acid $(0.3 \%, \mathrm{v} / \mathrm{v})$ and glucose $(0.1 \% \mathrm{w} / \mathrm{v})$ as carbon and energy sources. Similar results were obtained when the acid concentration was increased up to $0.5 \%$ $(\mathrm{v} / \mathrm{v})$. Although some strains, referred to as "slow strains", displayed a prolonged response time, this could be because those strains were more sensitive to the toxic effects of formic acid, yielding a lower growth rate in the mixed-substrate medium, and/or a reduced capability to transport and metabolise the acid. However, those strains displaying a faster alkalization must be considered the most troublesome in a beverage/food production plant. In the assays carried out using ZBD medium, several strains of Zygosaccharomyces bisporus were also included. Some of these strains exhibited a positive response, indicating that the medium developed is appropriate for the detection of both species. Z. bisporus has a similar spoilage potential to Z. bailii, but can be considered of rather less importance as wine spoilage yeast. Therefore, these results do not invalidate the application of the ZBD medium for the microbiological control of wines. With formic acid concentrations above $0.3 \%(\mathrm{v} / \mathrm{v})$, none of the other species showed an increase in the $\mathrm{pH}$ of the mixed-substrate culture media after $48 \mathrm{~h}$ of incubation, indicating lower tolerance of non-Z. bailii species to formic acid.

All the assays were carried out in liquid or solid medium. In industrial practice, the media most commonly used are solidified. As the results obtained with the liquid or solid mixed substrate media were analogous, the validation of the medium was performed with the latter. The percentage recovery of $Z$. bailii obtained with the ZBD medium was similar to that 
formerly described (1). ZBD was a better differential medium as it allowed a clear distinction between blue colonies formed by Z. bailii and colonies of other wine contaminating species like S. cerevisiae and P. membranaefaciens, facilitating the interpretation of results. The final composition of the ZBD medium (formic acid, $0.4 \% \mathrm{v} / \mathrm{v}$ and glucose, $0.1 \%$, w/v) was adjusted as a compromise between recovery and selectivity for Z. bailii.

ZBD medium (formic acid, $0.4 \% \mathrm{v} / \mathrm{v}$ and glucose, $0.1 \%$, w/v) was appropriate for the differential enumeration of Z. bailii in two samples of the same wine, originating from different wines (1 and 2). This species was only detected in wine 2 , suggesting the contamination occurred in the wine production plant. This is probably due to inadequate sanitation. The yeasts isolated from wine 2 and grown on WLD medium are most probably Z. bailii since the number of colonies is of the same order of magnitude as the blue colonies determined in the mixed-substrate medium. Nevertheless, in both wines analyzed, the ZBD medium recovered a higher number of yeast cells than WLD medium before refrigeration.

Refrigeration for four months caused a dramatic reduction of the total yeast count in the two samples. Under those conditions, Z. bailii colonies were not detected on ZBD. This may be attributed to the loss of cell viability or to an increased sensitivity to formic acid after long term storage under low temperature stress conditions. After the refrigeration period, the general medium for the isolation of yeasts (YEPD recovered a higher number of cells than WLN. Therefore, the former medium appears to be a more appropriate culture medium for the detection of yeast cells that have suffered stress during production and/or storage of wines.

The use of the newly described ZBD medium applying the membrane filtration method seems to be the most appropriate for two reasons. First, the white color of the membrane allows a good contrast to the blue colonies and therefore a better distinction of white or lightblue colonies formed by S. cerevisiae and blue colonies of Z. bailii, as illustrated in Fig. 1. Second, the low $\mathrm{pH}$ of the medium causes a reduced gel strength, so that inoculation by 
streaking or by spread-plating can be difficult. Nevertheless, the use of a sterile cotton swab instead of an inoculation loop for inoculation by streaking seems to be an acceptable solution.

Interestingly, this medium, with a percentage recovery around $60 \%$ for Z. bailii, allowed the detection of other wine contaminating yeast species. A differential medium able to recover most of the wine contaminating yeast might be achieved by the appropriate manipulation of the formic acid concentration.

In summary, the nature of the acid substrate, the manipulation of the $\mathrm{pH}$ and of the proportion of the two carbon and energy sources in the mixed medium allowed distinction of different behavior patterns among the various species tested. Based on such differences, and taking advantage of the simultaneous or sequential metabolism of the sugar and the acid a rapid method for the differentiation of $Z$. bailii was developed. The mixed-substrate medium ZBD allowed differential and simple detection of $Z$. bailii in contaminated wine samples. The inclusion of this medium in the group of media used for the microbiological monitoring of wine may allow the detection of $Z$. bailii, and consequently an adequate implementation of corrective measures when such a contamination is detected.

\section{Acknowledgments}

The authors want to express their gratitude to Dr. Virgílio Loureiro from the Departamento de Botânica e Engenharia Biológica of the Instituto Superior de Agronomia for the generous support regarding the confirmation of an isolated presumptive Z. bailii strain by molecular typing, for helpful discussions and the critical reading of this manuscript. This work was supported by the EU project AIR-2-CT93-830. 


\section{References}

1. Erickson J.P. 1993. Hydrophobic membrane filtration method for the selective recovery and differentiation of Zygosaccharomyces bailii in acidified ingredients. J. Food Prot., 563: 234-238.

2. Fernandes, M.L., M. Côrte-Real, V. Loureiro, M.C. Loureiro-Dias, and C. Leão. 1997. Glucose respiration and fermentation in Zygosaccharomyces bailii and Saccharomyces cerevisiae express different sensitivity patterns to ethanol and acetic acid. Lett. Appl. Microbiol. 25: 249-253.

3. Loureiro, V. 1994. Wine spoilage yeasts: a problem to be solved. "Assemblé General de l’Office International de la Vigne et du Vin”, Paris.

4. Makdesi, A.K., and L.R. Beuchat. 1996. Performance of selective media for enumerating Zygosaccharomyces bailii in acidic foods and beverages. J. Food Prot. 59: 652-656.

5. Sousa, M.J., L. Miranda, M. Côrte-Real, and C. Leão. 1996. Transport of acetic acid in Zygosaccharomyces bailii: effects of ethanol and their implications on the resistance of the yeast to acidic environments. Appl. Environ. Microbiol., 62: 3152-3157.

6. Sousa, M.J., F. Rodrigues, M. Côrte-Real, and C. Leão. 1998. Mechanism underlying the transport and intracellular metabolism of acetic acid in the presence of glucose in the yeast Zygosaccharomyces bailii. Microbiology, 144: 665-670.

7. Thomas, D.S., and R.R. Davenport. 1985. Zygosaccharomyces bailii - a profile of characteristics and spoilage activities. Food Microbiol., 2: 157-169.

8. van Uden, N. 1967. Transport-limited fermentation and growth of S. cerevisiae and its competitive inhibition. Arch. Microbiol. 58: 155-168.

\section{Patent}


Schuller, D., M. Côrte-Real, and C. Leão. May 1999. Meio de cultura para a detecção das leveduras Zygosaccharomyces bailii e Zygosaccharomyces bisporus. Instituto Nacional da Propriedade Intelectual (INPI), patent 12480 V. 


\section{Figure legends}

\section{Fig 1}

Morphology of the colonies of Zygosaccharomyces bailii in pure or mixed culture with other wine contaminating yeasts on membrane filters placed on the surface of the mixed-substrate medium containing formic acid $(0.2 \%, \mathrm{v} / \mathrm{v})$ and glucose $(0.1 \%, \mathrm{p} / \mathrm{v})$ after incubation for $96 \mathrm{~h}$ at $30^{\circ} \mathrm{C}$.
A Zygosaccharomyces bailii IGC $4806(\mathrm{Zb})$
B Saccharomyces cerevisiae IGC 4072 (Sc)
C Pichia membranaefaciens IGC $2487(P m)$
D Z. bailii IGC $4806(Z b)+$ S. cerevisiae IGC 4072 (Sc)
E Z. bailii IGC $4806(Z b)+$ P. membranaefaciens IGC $2487(P m)$ 


\section{Tables}

\section{TABLE 1}

Yeast strains used for the development of the differential culture medium.

Zygosaccharomyces bailii

Zygosaccharomyces bisporus

Zygosaccharomyces rouxii

Zygosaccharomyces florentinus

Debaryomyces hansenii

Dekkera anomala

Dekkera bruxellensis

Issatchenkia orientalis

Kloeckera apiculata

Kluyveromyces marxianus

Lodderomyces elongisporus

Pichia anomala

Pichia membranaefaciens

Rhodotorula mucilaginosa
IGC: 4267, 4806, 4227, 4531, T5167

ISA: 1023, 1024, 1025, 1031, 1095, 1148, 1206, $1212,1214,1265$

IGC: T5335, 5336, 5337, 5381, 5382, 5383, 5384, 5385

IGC: 4194, 3691, 3693, 3694, 3701

ISA: $1220,1552,1553$

IGC: 4169

IGC: 2968

INETI: CL18

IGC: 5133, 5160, 5161

IGC: 4179, 4801, 4808, 5162

IGC: 2631, 3341, 3806, 5041, 5044, 5046

ISA: 1189

IGC: 2671, 2902, 3014, 3286, 3886

ISA: 1308,1421

IGC: 2495, 2505, 3294, 4121, 4380, 4554, 5008

IGC: 2487, 2582, 3315, 3796, 4275, 4475, 4829, $4875,5013,5015,5017,5019,5122$

IGC: 4791, 5166 
Saccharomyces bayanus

Saccharomyces cerevisiae
IGC: 4565, T4456

CBS: 1871-1, 1871-2, 5494, 5495 
TABLE 1 (cont.)

Saccharomyces cerevisiae

Saccharomyces pastorianus

Saccharomycodes ludwigii

Schizosaccharomyces pombe

Torulaspora delbrueckii

$$
\begin{aligned}
& \text { IGC: T2608, 3507-III, 3507, 2917, 3970, 3977, } \\
& \quad \text { 4003, 4017, 4022, 4023, 4024, 4072, 4237, } \\
& \quad 4240,4241, \text { T4455, } 4891
\end{aligned}
$$

IGC: 4579, T4601

ISA: 1083, 1088, 1089

ISA: 1190, 1191, 1192, 1193

IGC: 4182, 2916, 3209, T2477

ISA: 1229, 1549

ISA Instituto Superior de Agronomia (Lisbon, Portugal)

IGC Instituto Gulbenkian de Ciências (Oeiras, Portugal)

INETI Instituto Nacional de Engenharia e Tecnologia Industrial (Lisbon, Portugal)

CBS Centraalbureau voor Schimmelcultures (Baarn, The Netherlands) 
TABLE 2

Alkaline response of different yeast species in liquid (LM) or solid (SM) mixed-substrate mineral medium containing glucose $(\mathrm{G}, 0.1 \% \mathrm{p} / \mathrm{v})$ and formic acid (FA, $0.3,0.4$ or $0.5 \%$, $\mathrm{v} / \mathrm{v})$ after $48 \mathrm{~h}$ of incubation at $30^{\circ} \mathrm{C}$.

\begin{tabular}{|c|c|c|c|c|c|c|c|}
\hline \multirow[t]{3}{*}{ Species } & Strain & \multirow{3}{*}{$\begin{array}{l}\mathrm{N}^{\mathrm{o}} \text { of } \\
\text { strains }\end{array}$} & \multirow{2}{*}{\multicolumn{2}{|c|}{$\begin{array}{l}\text { G0.1 } \\
\text { FA0.3 }\end{array}$}} & \multirow{2}{*}{\multicolumn{2}{|c|}{$\begin{array}{c}\text { G0.1 } \\
\text { FA0.4 }\end{array}$}} & \multirow{3}{*}{$\begin{array}{c}\text { G0.1 } \\
\text { FA0.5 } \\
\text { LM }\end{array}$} \\
\hline & Number * & & & & & & \\
\hline & & & $\mathrm{LM}$ & SM & $\mathrm{LM}$ & SM & \\
\hline \multirow[t]{4}{*}{ Zygosaccharomyces bailii } & & 12 & + & + & + & + & + \\
\hline & ISA 1025 & 1 & + & + & + & + & $-^{c}$ \\
\hline & ISA 1095 & 1 & + & $-a$ & $-b$ & $-b$ & $-^{c}$ \\
\hline & IGC T5167 & 1 & + & $-^{a}$ & $-b$ & $-b$ & $-^{c}$ \\
\hline \multirow[t]{2}{*}{ Zygosaccharomyces bisporus } & & 5 & + & + & + & + & n.d. \\
\hline & & 3 & + & + & $-{ }^{c}$ & $-^{c}$ & n.d. \\
\hline \multirow[t]{3}{*}{ Zygosaccharomyces rouxii } & & 6 & n.d. & n.d. & - & - & n.d. \\
\hline & IGC 4194 & 1 & + & + & $-{ }^{c}$ & $-{ }^{c}$ & - \\
\hline & ISA 1220 & 1 & - & - & $-^{c}$ & $-^{c}$ & - \\
\hline Zygosaccharomyces florentinus & & 1 & - & - & - & - & - \\
\hline Saccharomyces bayanus & & 2 & - & - & - & - & - \\
\hline Saccharomyces cerevisiae & & 21 & - & - & - & - & - \\
\hline Saccharomyces pastorianus & & 2 & - & - & - & - & - \\
\hline Saccharomycodes ludwigii & & 3 & n.d. & n.d. & - & - & n.d. \\
\hline Schizosaccharomyces pombe & & 4 & n.d. & n.d. & - & - & n.d. \\
\hline Pichia membranaefaciens & & 13 & - & - & - & - & - \\
\hline
\end{tabular}


TABLE 2 (cont.)

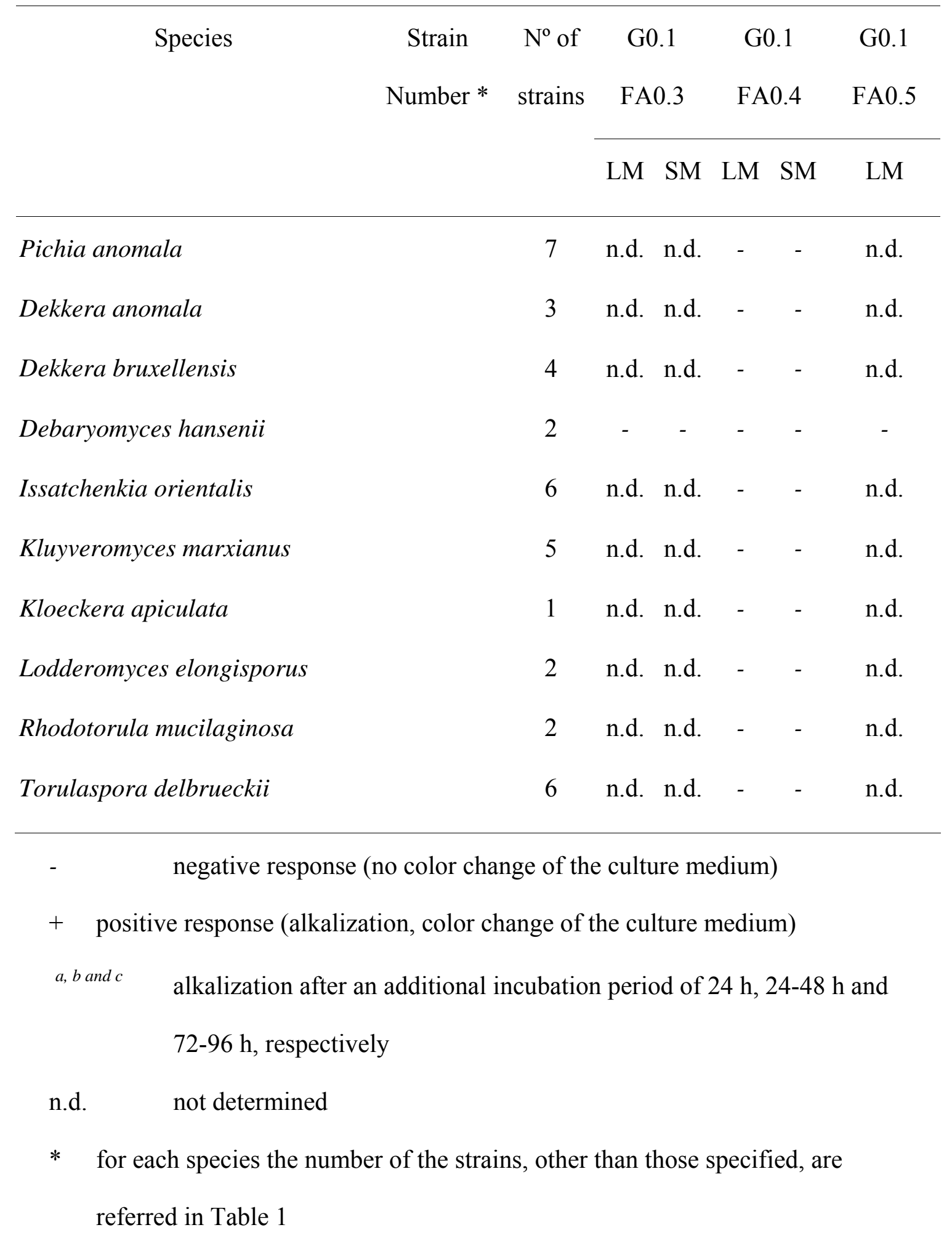


TABLE 3. Percentage recovery (\%) of Zygosaccharomyces bailii cells in pure or mixed culture with other wine contaminating yeast species on Zygosaccharomyces bailii Selective Agar (ZBA) and on Zygosaccharomyces bailii Differential (ZBD) medium with different formic acid concentrations, using YEPD as reference medium, after incubation for $96 \mathrm{~h}$ at $30^{\circ} \mathrm{C}$.

\begin{tabular}{|c|c|c|c|c|c|c|c|}
\hline \multicolumn{2}{|l|}{ Strain(s) } & \multirow{3}{*}{ YEPD } & \multirow{3}{*}{$\mathrm{ZBA}$} & \multicolumn{4}{|c|}{ ZBD Culture medium } \\
\hline & & & & G0.1 & G0.1 & G0.1 & G0.1 FA0.气 \\
\hline & & & & FA0.2 & FA0.3 & FA0.4 & \\
\hline Z. bailii & IGC 4806 & 100 & 72 & 82 & 78 & 65 & $42^{a}$ \\
\hline Z. bailii + & IGC 4806 & 100 & 77 & 82 & 81 & 57 & $35^{a}$ \\
\hline S. cerevisiae & IGC 4072 & n.d. & n.d. & n.d. & n.d. & n.d. & n.d. \\
\hline Z. bailii + & IGC 4806 & 100 & 82 & 99 & 94 & 67 & $34^{a}$ \\
\hline P. membranaefaciens + & IGC 2487 & n.d. & n.d. & n.d. & n.d. & n.d. & n.d. \\
\hline D. anomala & IGC 5133 & n.d. & n.d. & n.d. & n.d. & n.d. & n.d. \\
\hline
\end{tabular}

$\begin{array}{lccccccc}\text { S. cerevisiae } & \text { IGC 4072 } & 100 & 6^{a} & 30^{a} & 4^{a} & <0.002^{a} & <0.002^{a} \\ \text { P. membranaefaciens } & \text { IGC 2487 } & 100 & <0.004^{a} & 55^{a} & 5.9^{a} & 0.011^{a} & <0.004^{a} \\ \text { D. anomala } & \text { IGC 5133 } & 100 & <0.004^{a}<0.004^{a}<0.004^{a}<0.004^{a}<0.004^{a}\end{array}$

G0.1FA0.2, G0.1FA0.3, G0.1FA0.4 and G0.1FA0.5: Mineral medium containing glucose $(0.1 \%, \mathrm{w} / \mathrm{v})$ and formic acid $(0.2,0.3,0.4$ or $0.5 \%, \mathrm{v} / \mathrm{v})$, respectively

a significant difference $(\mathrm{p} \leq 0.05)$ compared to the reference culture medium (YEPD)

n.d. not determined 
TABLE 4

Analysis of two samples of contaminated "Vinho Verde" using the mixed-substrate culture medium ZBD (FA0.4 G0.1) containing formic acid (0.4\%, v/v) and glucose $(0.1 \%$, v/v) and the media Yeast Extract Peptone Dextrose (YEPD), Wallerstein Laboratory Nutrient (WLN) and Differential (WLD). The incubation was carried out at $30^{\circ} \mathrm{C}$ for $96 \mathrm{~h}$ and the results expressed in CFU per $\mathrm{ml}$ of wine.

\begin{tabular}{cccccc}
\hline & \multicolumn{2}{c}{ Wine 1} & & \multicolumn{2}{c}{ Wine 2 } \\
\cline { 2 - 3 } \cline { 5 - 6 } Culture medium & before & after & & before & after \\
& refrigeration & refrigeration & & refrigeration & refrigeration \\
n.d. & 685 & 107 & & n.d. & 85 \\
YEPD & 10 & 73 & & 620 & 2 \\
WLN & $75^{1}$ & 3 & & 200 & 2 \\
ZBD: FA0.4 G0.1 & & 0 & $90^{1}+170^{2}$ & 0 \\
\hline
\end{tabular}

1 slightly yellow, creamy colonies

2 blue colonies, typical of Z. bailii

n.d. not determined 

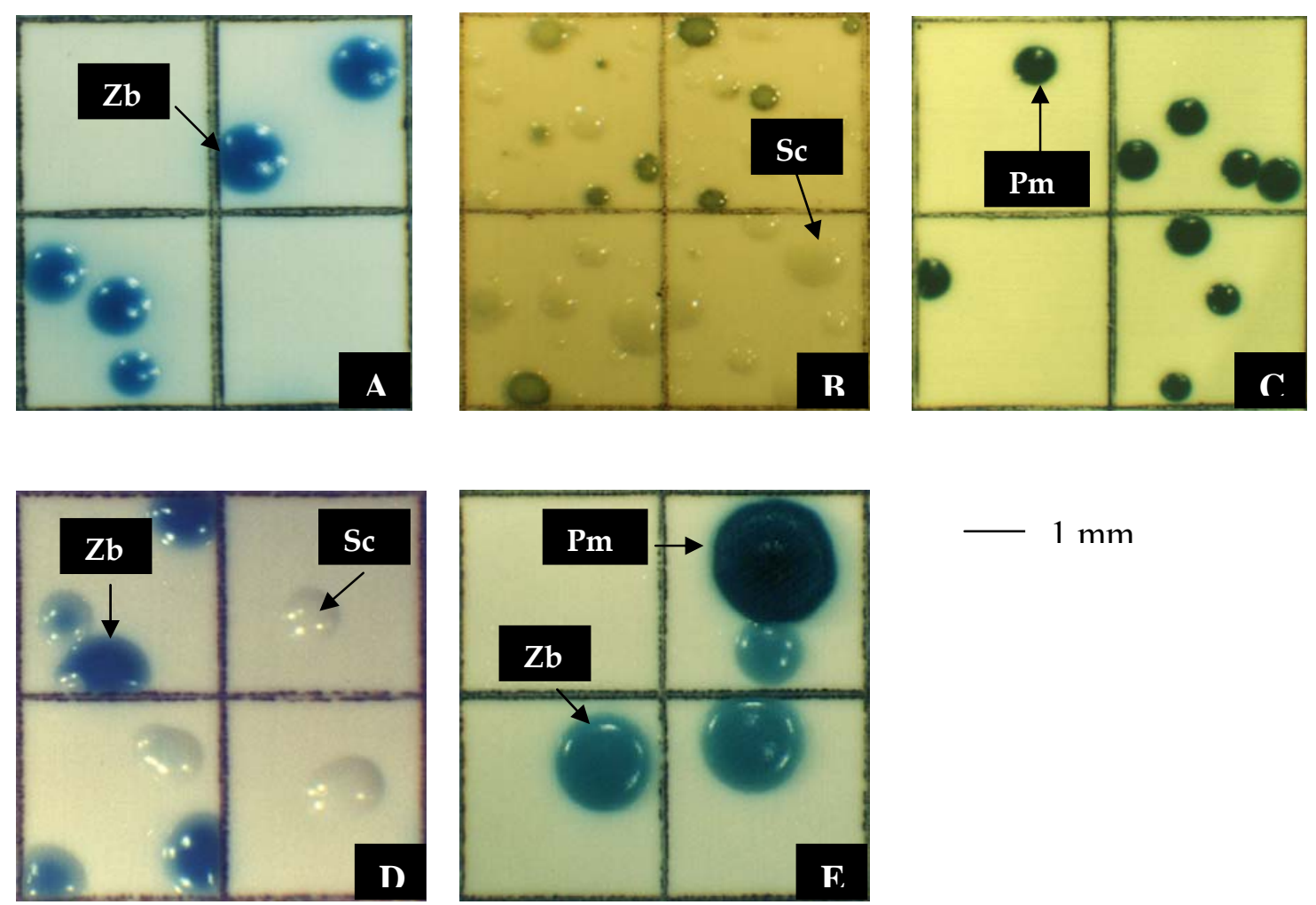